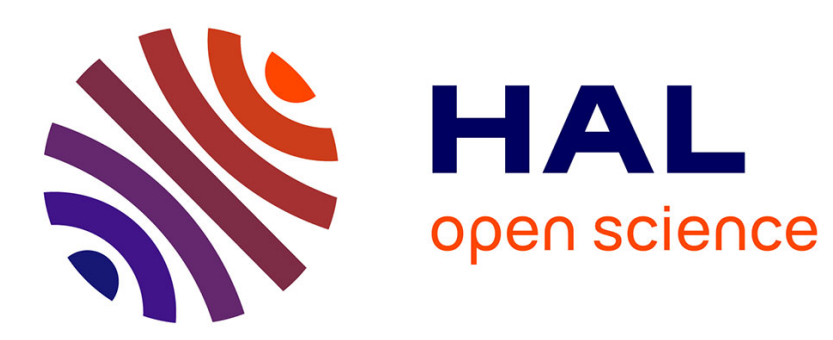

\title{
Turbo Detection Based On Sparse Decomposition For Massive MIMO Transmission
}

\author{
Zahran Hajji, Karine Amis Cavalec, Abdeldjalil Aissa El Bey
}

\section{To cite this version:}

Zahran Hajji, Karine Amis Cavalec, Abdeldjalil Aissa El Bey. Turbo Detection Based On Sparse Decomposition For Massive MIMO Transmission. ISTC 2016: 9th International symposium on Turbo Codes and Iterative Information Processing, Sep 2016, Brest, France. pp.1 - 5. hal-01379489

\author{
HAL Id: hal-01379489 \\ https://hal.science/hal-01379489
}

Submitted on 11 Oct 2016

HAL is a multi-disciplinary open access archive for the deposit and dissemination of scientific research documents, whether they are published or not. The documents may come from teaching and research institutions in France or abroad, or from public or private research centers.
L'archive ouverte pluridisciplinaire HAL, est destinée au dépôt et à la diffusion de documents scientifiques de niveau recherche, publiés ou non, émanant des établissements d'enseignement et de recherche français ou étrangers, des laboratoires publics ou privés. 


\title{
Turbo Detection Based On Sparse Decomposition For Massive MIMO Transmission
}

\author{
Zahran Hajji, Karine Amis and Abdeldjalil Aïssa-El-Bey \\ Institut Mines-Telecom, Telecom Bretagne, Lab-STICC, UMR CNRS 6285, 29238 Brest, France \\ Université européenne de Bretagne \\ Email: Firstname.Lastname@ @elecom-bretagne.eu
}

\begin{abstract}
In this paper, we address the problem of underdetermined massive MIMO detection (the number of observations is less than the number of sources) assuming QAM constellations. In [1], the authors showed the utility of projecting the signal on a basis of the modulation alphabet, looking for the sparsest vector representation. As an extension of this work and in order to reduce the detection complexity, we present first an equivalent real-valued formulation of the optimization problem, all the more interesting as the modulation order is high. Then we consider an outer forward error correcting (FEC) code and we propose a turbo detection scheme. We focus on the medium SNR value range where detection errors involve adjacent symbols. Based on this hypothesis, we propose a sparse vector formulation to be treated as a soft detection output that can be directly exploited in a symbol-to-binary conversion to feed the FEC decoder with reliable soft input. The FEC decoder output will be exploited to provide a priori information within the detection criterion based on a regularization approach. Simulation results show the efficiency of the proposed scheme in comparison with reference schemes of the state-of-art.
\end{abstract}

Index Terms-sparse representation, source separation, massive MIMO, turbo-detection

\section{INTRODUCTION}

$\mathrm{E}^{\mathrm{x}}$ XPONENTIAL growth in the number of mobile connected machines and the amount of data they consume motivate researchers to look for new technologies and approaches to address the mounting demand. MIMO technology has been selected in the $5 \mathrm{G}$ standard definition as a solution to provide higher throughput under spectrum limitations [2]. It promises significant gains that offer the ability to serve more users at higher data rates with better reliability. Large number of antennas and/or users is involved, which makes the receiver design critical from the complexity point of view. Sphere decoding technique which is a Maximum Likelihood (ML) based detector involves an exhaustive search within the hypersphere whose dimensions remain high in the large-scale MIMO case, yielding computationally-unsolvable detection. Research for high-performance receiver design that can lead to practical realization of large-MIMO systems is both nascent as well as promising [3]. Usual linear equalizers such as minimum mean square error (MMSE) and zero-forcing ( $\mathrm{ZF}$ ) have low computation complexity but perform poorly when used in underdetermined uncoded MIMO systems. Such configuration

The authors would like to thank the Brittany Region and PRACOM for their financial support. is expected in future $5 \mathrm{G}$ system uplink, as the number of connected users times their transmit antenna number could be much higher than the base station receive antenna number.

Previous works proved that source separation is possible in the underdetermined case thanks to basis pursuit (BP) techniques. Following this approach, a sparse representation was proposed in [4] to define a successful separation method for a conditioned dimension system. Underdetermined noisy MIMO system with finite alphabet was dealt with as an application case of [4] in [1] and [5], where the problem was formulated as a basis pursuit denoising (BPDN) problem with relaxed constraints. This formulation is based on an ML criterion applied with relaxed constraints.

In this paper, we consider the method developed in [1] and we investigate its complexity reduction and its use in association with a FEC decoder. The contributions are threefold. The first one is a reduced-complexity detection with error rate performance preservation thanks to a real-valued formulation of the problem. The second one is the definition of reliable soft detection output to be delivered to a FEC decoder. Thirdly we propose an iterative receiver based on a regularization procedure in the detection optimization criterion.

This paper is organized as follows. Section II describes the underdetermined MIMO transmission model. Section III deals with the source separation problem in the MIMO case. We consider a real-valued formulation of the problem and propose a new sparse decomposition. Section IV describes how the proposed sparse decomposition-based detector is adapted to exchange soft information with a FEC decoder. In Section $\mathrm{V}$, we compare the proposed sparse decomposition-based detection method to the one investigated in [1] as well as to the MMSE linear equalizer in both coded and uncoded cases. Finally, Section VI concludes the paper.

Notations: boldface upper case letters and boldface lower case letters denote matrices and vectors, respectively. For the transpose, transpose conjugate and conjugate matrices we use $(.)^{T},(.)^{H}$ and (.)* , respectively. $\otimes$ is the Kronecker product. $\boldsymbol{I}_{p}$ is the $p \times p$ identity matrix and $\mathbf{1}_{p}$ is the all-one size- $p$ vector.

\section{SYSTEM MODEL}

Let us consider a $K$-user MIMO transmission over a flat fading channel, where each user has $N_{t}$ transmit antennas and 
let $N=K \times N_{t}$. At the base station side, we assume $n$ equivalent receive antennas (base station cooperation through the base station controller is possible) with $n \leq N$. We assume a perfect knowledge of the channel state information (CSI) at the base station. In this paper, we focus on the uplink of the communication system. The received signal is defined as follows:

$$
\boldsymbol{y}=\boldsymbol{H} \boldsymbol{x}+\boldsymbol{z} .
$$

where $\boldsymbol{H}$ is an $n \times N$ random channel matrix, $\boldsymbol{x}$ is the $N \times 1$ data vector, and $\boldsymbol{z}$ is the $n \times 1$ complex circularly symmetric additive Gaussian noise vector with zero mean and a covariance matrix equal to $\sigma^{2} \boldsymbol{I}$. We assume that the components of $\boldsymbol{x}$ belong to an $M$-QAM modulation alphabet such that $M=p^{2}$ (square QAM constellation). The modulation alphabet is denoted by $\mathcal{Q}=\left\{q_{1}, q_{2}, \ldots, q_{M}\right\}$. We associate to $Q$ the symbol vector $\boldsymbol{q}=\left[q_{1}, q_{2}, \ldots, q_{M}\right]$ where $q_{i}=a_{i}+j b_{i}$, $i \in\{1, . ., . M\}$ with $\left(a_{i}, b_{i}\right) \in \mathcal{A} \times \mathcal{A}$ and $\mathcal{A}=\left\{\alpha_{1}, \alpha_{2}, . ., \alpha_{p}\right\}$.

The challenge is to design an efficient joint detection scheme of the transmitted data with moderate computational complexity order over the whole SNR range in an underdetermined system $(n \leq N)$ and to introduce it within an iterative turbo-like detection receiver.

\section{ITERATIVE DETECTION OF SPARSE DECOMPOSED MIMO SIGNAL VIA EUCLIDEAN DISTANCE MINIMIZATION}

\section{A. Proposed detection scheme}

We now consider the MIMO system described in Section II with large values of $n$ and $N$ (massive MIMO configuration). In this respect, the optimal ML joint detection, based on exhaustive search is a problem computationally unsolvable. Using the decomposition proposed in [1], the received signal reads

$$
\boldsymbol{y}=\boldsymbol{H} \boldsymbol{B}_{q} \boldsymbol{r}+\boldsymbol{z},
$$

where $\boldsymbol{B}_{q}=\boldsymbol{I}_{N} \otimes \boldsymbol{q}$ and $\boldsymbol{q}$ is the complex-valued alphabet vector $\boldsymbol{q}=\left[q_{1}, q_{2}, \ldots, q_{M}\right]$.

The vector $\boldsymbol{r}$ is sparse as it is the concatenation of $N$ subvectors, each with only one non-null component. The nonzero component equals one and corresponds to the transmitted symbol (.i.e. if $r_{\ell N+k}=1$, then the $(\ell+1)$-th user symbol equals $\left.q_{k}\right)$.

In order to recover the sparse vector $\boldsymbol{r}$, the authors in [4] proposed to solve the following optimization problem:

$$
\begin{aligned}
\left(P_{\mathcal{C}, 2}\right): \underset{\boldsymbol{r}}{\arg \min } & \left\|\boldsymbol{y}-\boldsymbol{H} \boldsymbol{B}_{q} \boldsymbol{r}\right\|_{2} \\
\text { subject to } \boldsymbol{B}_{1}^{\mathrm{e}} \boldsymbol{r} & =\mathbf{1}_{N}, \\
\boldsymbol{r} & \geq 0 .
\end{aligned}
$$

where $\boldsymbol{B}_{1}^{\mathcal{C}}=\boldsymbol{I}_{N} \otimes \mathbf{1}_{M}$.

This optimization problem statement can be seen as a ML relaxed detector that minimizes the Euclidean distance with the received signal. The constraints are invoked to impose the sparse structure of the vector $\boldsymbol{r}$.

As we can see the complexity of this proposed procedure increases with the constellation size $M$. To get around this main drawback we transform first the complex-valued system into an equivalent real-valued system, which reads

$$
\tilde{\boldsymbol{y}}=\tilde{\boldsymbol{H}} \tilde{\boldsymbol{x}}+\tilde{\boldsymbol{z}},
$$

where $\tilde{\boldsymbol{y}}=\left(\begin{array}{l}\Re y \\ \Im y\end{array}\right), \tilde{\boldsymbol{H}}=\left(\begin{array}{cc}\Re \boldsymbol{H} & -\Im \boldsymbol{H} \\ \Im \boldsymbol{H} & \Re \boldsymbol{H}\end{array}\right), \tilde{\boldsymbol{x}}=\left(\begin{array}{c}\Re x \\ \Im \boldsymbol{x}\end{array}\right)$ and $\tilde{z}=\left(\begin{array}{c}\Re z \\ \Im z\end{array}\right)$.

In this real-valued system, the first $N$ components of $\tilde{\boldsymbol{x}}$ are the real parts of the symbols and the imaginary parts are mapped to the next $N$ components. Exploiting the same approach as in [1] and based on the fact that the elements of $\tilde{\boldsymbol{x}}$ belong to the real-valued alphabet $\mathcal{A}=\left\{\alpha_{1}, \alpha_{2}, . ., \alpha_{p}\right\}$ we decompose $\tilde{\boldsymbol{x}}$ as $\tilde{\boldsymbol{x}}=\boldsymbol{B}_{\alpha} \boldsymbol{s}$ where $\boldsymbol{B}_{\alpha}=\boldsymbol{I}_{N} \otimes \boldsymbol{\alpha}$, with $\boldsymbol{\alpha}=\left[\alpha_{1}, \alpha_{2}, \ldots, \alpha_{p}\right]$. The vector $\boldsymbol{s}$ is sparse. Each of its $2 N$ subvectors contains one element different from 0 that corresponds to the equivalent real symbol. Then, we propose to recover $\boldsymbol{x}$ from the following optimization procedure:

$$
\begin{aligned}
\underset{\left.P_{\mathcal{R}, 2}\right): \underset{s}{\arg \min }}{ } & \left\|\tilde{\boldsymbol{y}}-\tilde{\boldsymbol{H}} \boldsymbol{B}_{\alpha} \boldsymbol{s}\right\|_{2} \\
\text { subject to } \boldsymbol{B}_{1}^{\mathcal{R}} \boldsymbol{s} & =\mathbf{1}_{2 N}, \\
\boldsymbol{s} & \geq 0 .
\end{aligned}
$$

where $\boldsymbol{B}_{1}^{\mathcal{R}}=\boldsymbol{I}_{2 N} \otimes \mathbf{1}_{p}$.

\section{B. Soft-decision detection output}

In the noisy case, there exist many decompositions satisfying the optimization constraints and yielding the same $\hat{s}$. In some cases, the detection algorithm converges to the true one: $\hat{\tilde{\boldsymbol{x}}}$ is sparse and its subvectors have only one nonnull component which equals 1 . Otherwise, the decomposition is erroneous and $\hat{\tilde{\boldsymbol{x}}}$ is not sparse. To ensure a reliable soft detection output, we try to obtain a decomposition estimate of $\tilde{\boldsymbol{x}}$ as close as possible to the true one. To this end, we focus on SNR values such that, when an error occurs, the detected symbol is an adjacent symbol. Then we can transform each non-sparse subvector into a sparse one with only two non-null components corresponding to the alphabet elements which are closed to the original estimate. Let $\widehat{\widetilde{\boldsymbol{x}}}_{j}=\boldsymbol{\alpha}^{T} \hat{\boldsymbol{s}}_{j}$ be an estimation of $j$-th element of the searched vector where $\hat{s}_{j}$ is non-sparse. We first find the index $i$ such that the real symbols $\alpha_{i}$ and $\alpha_{i+1}$ are the closest to $\widehat{\tilde{\boldsymbol{x}}}_{j}$. We then force all the components of $\hat{\boldsymbol{s}}_{j}$ to 0 except $\hat{\boldsymbol{s}}_{j}(i)=\frac{\widehat{\widehat{\boldsymbol{x}}}_{j}-\alpha_{i+1}}{\alpha_{i}-\alpha_{i+1}}$ and $\hat{\boldsymbol{s}}_{j}(i+1)=1-\hat{\boldsymbol{s}}_{j}(i)$.

\section{TURBO SPARSE DECOMPOSITION-BASED DETECTION}

\section{A. Iterative receiver and soft-output detection}

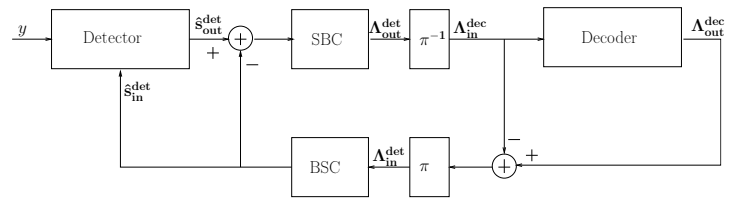

Fig. 1: Turbo detection scheme. 
Our goal is to associate the proposed soft-output detector with a FEC decoder and make them work in an iterative manner (see Fig. 1). We now consider that the binary stream is FEC encoded, then randomly interleaved before being converted into QAM symbols and passed through a serialto-parallel converter.

Back to our model, each real or imaginary parts of the symbol are seen as a projection of a sparse sub-vector onto the finite real-valued alphabet vector $\boldsymbol{\alpha}$. To satisfy the constraints in the noiseless case, each subvector must have only one nonzero component equal to one at the position of the associated value in $\boldsymbol{\alpha}$. In the noisy case, the detection output satisfies the optimization constraints: the subvector components are positive and their sum equals 1 . Each $j$-th subvector component can be interpreted as the probability that the real part (or imaginary part) be equal to $\alpha_{j}$. This interpretation is used to build the soft detection output to be delivered to the FEC decoder. We consider in the following each recovered element in the interval $[0,1]$ as the probability of a symbol to occur conditionally to $\boldsymbol{y}$.

Let $m=\log _{2}(\sqrt{M})$ and $\mathbf{c}$ be the length- $m N$ coded and interleaved binary information sequence at one channel use. Let also $\psi$ be the binary-to-symbol conversion defined as:

$$
\psi:\left[c_{(k-1) m} c_{(k-1) m+1} \ldots c_{k m-1}\right] \in\{0,1\}^{m} \mapsto \tilde{x}_{k} \in \mathcal{A}
$$

and $c_{j}=\psi^{-1}\left(\alpha_{j}\right)$.

At first iteration, the sparse detector provides $\hat{s}_{\text {out }}^{\text {det }}$ interpreted as a posteriori probabilities of $\tilde{x}$ :

$$
\hat{\boldsymbol{s}}_{\text {out }}^{\text {det }}((k-1) m+j)_{=}^{\Delta} \operatorname{Pr}\left(\tilde{x}_{k}=\alpha_{j} \mid y\right)
$$

Using $\hat{s}_{\text {out }}^{\text {det }}$, the symbol to binary converter (SBC) computes the $\log$ likelihood ratio on the $i$-th bit associated to the $k$-th symbol, denoted by $\Lambda_{\text {out }}^{\text {det }}$ and defined as:

$$
\begin{aligned}
\Lambda_{\text {out }}^{\text {det }}((k-1) m+i) & =\log \left(\frac{\operatorname{Pr}\left(c_{(k-1) m+i}=1 \mid y\right)}{\operatorname{Pr}\left(c_{(k-1) m+i}=0 \mid y\right)}\right) \\
& =\log \left(\frac{\sum_{\alpha_{j} \in \mathcal{A}_{i, 1}} \operatorname{Pr}\left(\tilde{x}_{k}=\alpha_{j} \mid y\right)}{\sum_{\alpha_{j} \in \mathcal{A}_{i, 0}} \operatorname{Pr}\left(\tilde{x}_{k}=\alpha_{j} \mid y\right)}\right) \\
& =\log \left(\frac{\sum_{\alpha_{j} \in \mathcal{A}_{i, 1}} \hat{\boldsymbol{s}}_{\text {out }}^{\text {det }}((k-1) m+j)}{\sum_{\alpha_{j} \in \mathcal{A}_{i, 0}} \hat{\boldsymbol{s}}_{\text {out }}^{\text {det }}((k-1) m+j)}\right)
\end{aligned}
$$

with $\mathcal{A}_{i, \epsilon}=\left\{a \in \mathcal{A} \mid \psi^{-1}(a)(i)=\epsilon\right\}$.

Let $\Lambda_{i n}^{\text {dec }}$ be the sequence obtained after deinterleaving of $\Lambda_{\text {out }}^{\text {det }}$. We consider that the FEC code is a convolutional code and assume that the soft-in soft-out optimal Bahl Cocke Jelinek Raviv (BCJR) decoder [6] is used at the receiver. The FEC decoder produces $\Lambda_{\text {out }}^{\text {dec }}$. It can be decomposed as the sum of $\Lambda_{i n}^{d e c}$ and $\Lambda_{e x t}^{d e t}$. $\Lambda_{e x t}^{d e t}$ is extrinsic information, used as input of the binary-to-symbol converter (BSC) to provide a priori information to the detector in the following iteration. Let $\Lambda_{i n}^{\text {det }}$ be the result of interleaving of $\Lambda_{e x t}^{d e c}$. The detector a priori information is denoted by $\hat{s}_{i n}^{\text {det }}$ and reads

$$
\begin{aligned}
\hat{s}_{i n}^{\text {det }}((k-1) m+j) & =\operatorname{Pr}\left(\tilde{\boldsymbol{x}}_{\boldsymbol{k}}=\alpha_{j} \mid \Lambda_{i n}^{\text {det }}\right) \\
& =\prod_{\substack{0 \leq i \leq m-1 \\
c_{j}=\psi^{-1}\left(\alpha_{j}\right)}} \operatorname{Pr}\left(c_{(k-1) m+i}=c_{j}(i) \mid \Lambda_{i n}^{\text {det }}\right)
\end{aligned}
$$

with $\operatorname{Pr}\left(c_{(k-1) m+i}=c_{j}(i) \mid \Lambda_{\text {in }}^{\text {det }}\right)=\frac{\exp (u v)}{\exp (v)+\exp (-v)}$ with $u=2 c_{j}(i)-1$ and $v=\frac{\Lambda_{i n}^{\text {det }}((k-1) m+i)}{2}$.

\section{B. Detection criterion}

In order to take into account the a priori information delivered by the decoder in the proposed detector, we propose to resolve the optimization system with a regularization term as follows:

$$
\left(P_{\mathcal{R}, 2}\right): \underset{s}{\arg \min }\left\|\hat{\boldsymbol{y}}-\hat{\boldsymbol{H}} \boldsymbol{B}_{\alpha} \boldsymbol{s}\right\|_{2}+\gamma\left\|\boldsymbol{s}-\hat{\boldsymbol{s}}_{i n}^{\text {det }}\right\|_{2}
$$

where $\gamma$ is a positive weight less than 1. It enables to take into account the unaccuracy of the estimated vector. The second term of regularization can be seen as a penalty, imposed to ensure that the detector output remains in the neighborhood of the decoder output.

\section{Choice of the regularization parameter}

The performance of our proposed detector depends highly on the choice of the regularization parameter. We propose two approaches. The first considers $\gamma$ constant for all the iterations. The parameter is then found empirically for each SNR value. The second makes the parameter value change over the iterations. In other words, for each SNR value, $\gamma$ will depend on the probability that $\hat{\boldsymbol{s}}_{i n}^{\text {det }}$ is the searched vector which can be simply determined with the LLR provided by the decoder.

\section{Simulation RESUlts}

In this section, we evaluate the new real-valued sparse decomposition-based detection both in uncoded and coded cases in terms of complexity and error rate compared to stateof-art techniques. The channel coefficients are assumed to be i.i.d circularly symmetric complex Gaussian distributed with zero mean and unit variance. We use the CVX toolbox, which is a Matlab library for convex optimization [7]. The quadratic minimization problem is solved by the Gurobi optimizer [8]. The simulation results are obtained by using a PC with OS Linux Ubuntu 14.04 with processor Intel Core i3-2350M 2.3 $\mathrm{GHz}$ and $8 \mathrm{~GB}$ of RAM memory.

\section{A. Complexity analysis}

The interest of the proposed real-valued transformation comes from its complexity order. The CVX toolbox relies on the interior point method whose complexity is a function of the number of constraints and the dimension of the searched vector. A convex optimization problem defined over $\mathbb{R}^{m}$ under $d$ constraints requires, in the worst case, $O(\sqrt{d})$ iterations for a computation cost of order $O\left(m^{2} d\right)$ per iteration and yields a total computation cost of order $O\left(m^{2} d^{3 / 2}\right)$ [9]. Applied to $\left(P_{\mathcal{C}, 1}\right)$ and $\left(P_{\mathcal{R}, 1}\right)$, the complexity ratio equals $\sqrt{M}$. The 


\begin{tabular}{|r|c|c|l|}
\hline & Iteration number & Computational cost per iteration & Total \\
\hline$\left(P_{\mathcal{C}, 1}\right)$ & $O(M \sqrt{N})$ & $O\left(N^{2.5}\right)$ & $O\left(M N^{3}\right)$ \\
\hline$\left(P_{\mathcal{R}, 1}\right)$ & $O(\sqrt{M N})$ & $O\left(N^{2.5}\right)$ & $O\left(\sqrt{M} N^{3}\right)$ \\
\hline$M M S$ & 1 & $O\left(n^{3}\right)$ & $O\left(n^{3}\right)$ \\
\hline$S D$ & 1 & $O\left(\sqrt{M^{N}}\right)$ & $O\left(\sqrt{M^{N}}\right)$ \\
\hline
\end{tabular}

TABLE I: Computational cost with the interior point method.

computational costs are reported in Table I. The proposed realvalued problem $\left(P_{\mathcal{R}, 2}\right)$ is all the more interesting to solve as the modulation order is high. The sphere-detection is NPhard [10]: thus it is the least cost efficient. The MMSE-based detector involves a complexity with the order of $O\left(n^{3}\right)$. In the case of low-order modulation and determined system, the proposed detector complexity is of the same order as the MMSE one.

\section{B. Non-Coded case}



Fig. 2: BER performance comparison with $N=16$ and $n=$ 14 and 4-QAM (uncoded).

Fig. 2 gives the BER performance of the proposed $\left(P_{\mathcal{R}, 2}\right)$ detector, the sphere decoder (SD), and the $\left(P_{\mathcal{C}, 2}\right)$ detector in the uncoded case with $N=16, n=14$ and $M=4$. As $\left(P_{\mathfrak{C}, 2}\right)$ and $\left(P_{\mathcal{R}, 2}\right)$ resort to the same detection principle, they perform the same whatever the system configuration. Beyond $6.7 \mathrm{~dB}$, the SD outperforms the $\left(P_{\mathcal{R}, 2}\right)$ detector, e.g. at BER $10^{-3}$, a gain of about $5 \mathrm{~dB}$ is achieved.

In Fig. 4, the system dimension is increased to $64 \times 64$ (determined). The SD complexity becomes too high. We thus compare the $\left(P_{\mathcal{R}, 2}\right)$ detector to the MMSE detector (which requires $N \leq n)$. One can observe that the $\left(P_{\mathcal{R}, 2}\right)$ detector better exploits the receive diversity than the MMSE detector. At BER $10^{-2}$, the $\left(P_{\mathcal{R}, 2}\right)$ outperforms the MMSE by about $5.5 \mathrm{~dB}$. This gain increases and exceeds $7 \mathrm{~dB}$ for a BER of $10^{-3}$.

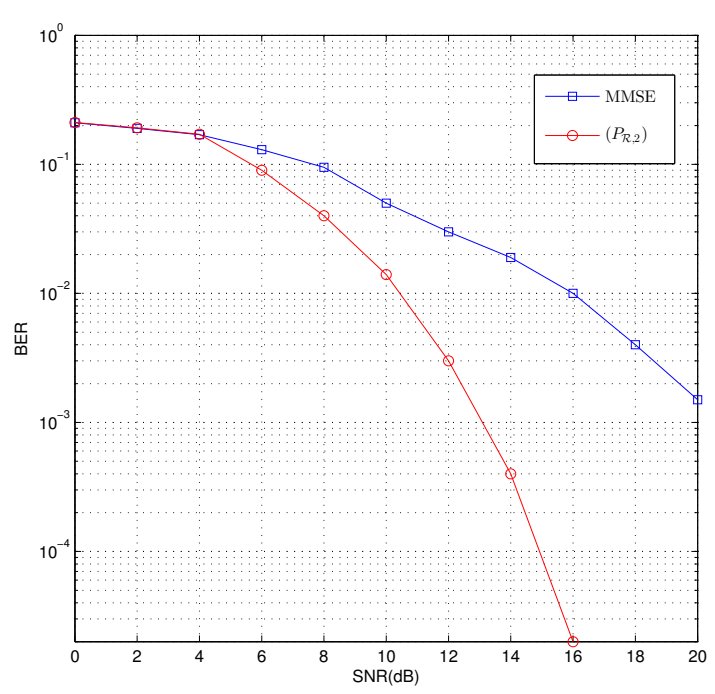

Fig. 3: BER performance comparison with $N=64$ and $n=$ 64 and 4-QAM (uncoded).

\section{Coded case}

In this section, we compare the proposed turbo detection scheme to a MMSE-based turbo equalizer [11]. The convolutional code $(\mathrm{CC})$ polynomials in octal are $(13,15)$ with a code rate equal to 0.5 . We take into account a variable regularization parameter $\gamma$ in the detection criterion.

In Fig. 4, we consider $N=128, n=96, L=512$ (frame length) and $M=4$. We observe that the proposed scheme outperforms the turbo-MMSE scheme for all the number of iterations. It can be seen that the gain increases with the SNR value. For instance, it achieves $1 \mathrm{~dB}$ at $\mathrm{BER}=10^{-4}$ at the fourth iteration.

In Fig. 5 , we consider $N=16, n=15, L=512, M=$ 16 and 4 iterations. The efficiency of the exploitation of the extrinsic information between the detector and the decoder can be shown by looking at the improvement from an iteration to the next one. For example, from the second iteration to the third one, the gain is of about $3 \mathrm{~dB}$ at $\mathrm{BER}=10^{-3}$.

As mentioned above in Section IV-C, the regularization parameter in the detector can be fixed by two approaches. Fig. 6 compares the two methods for $N=n=64, L=512$ and $M=4$. The first one takes $\gamma=1 / 3$. The second one makes $\gamma$ variable and crescent as the number of iterations increases, that is to say as the FEC output gets more reliable. We mention that the values are optimized empirically. Simulations show that both approaches converge to the same performance as 
observed in Fig. 6 but for the intermediate iterations, the probabilistic choice outperforms the deterministic (fixed) one.

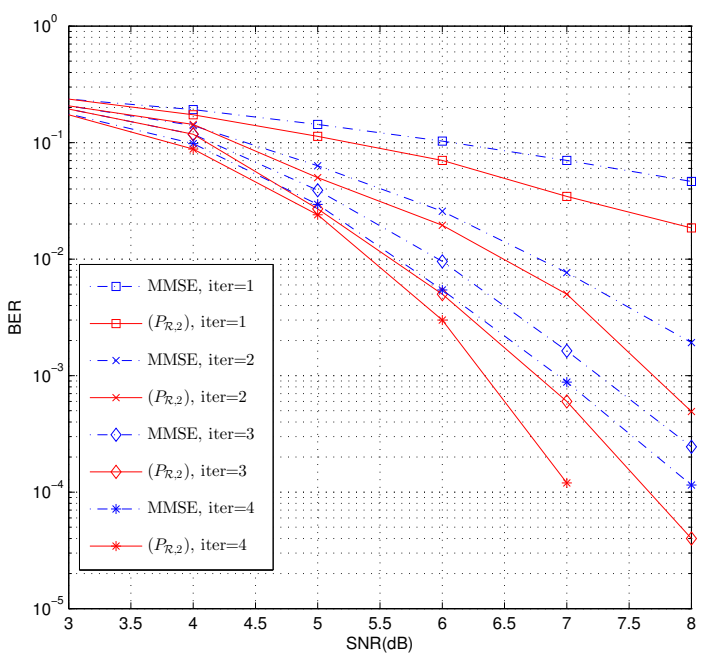

Fig. 4: BER performance comparison with $N=128, n=96$, $L=512, \mathrm{CC}(13,15)$ and 4 -QAM (iterative receiver).

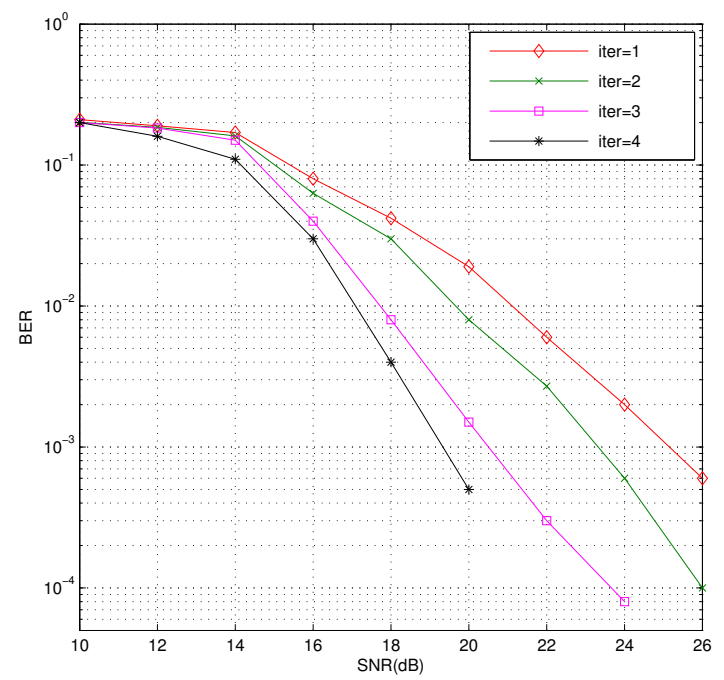

Fig. 5: BER performance comparison with $N=16, n=15$, $L=512, \mathrm{CC}(13,15)$ and 16-QAM BER (iterative receiver).

\section{CONCLUSION}

In this paper we have addressed the problem of detection in large MIMO systems with finite $M$-QAM constellation. We have proposed a real-valued formulation of the system model and then we have decomposed each symbol sparsely in the real-valued alphabet basis. Exploiting the vector sparsity and following the approach of [1], we have defined the detection based on the problem $\left(P_{\mathcal{R}, 2}\right)$ which achieves the same error

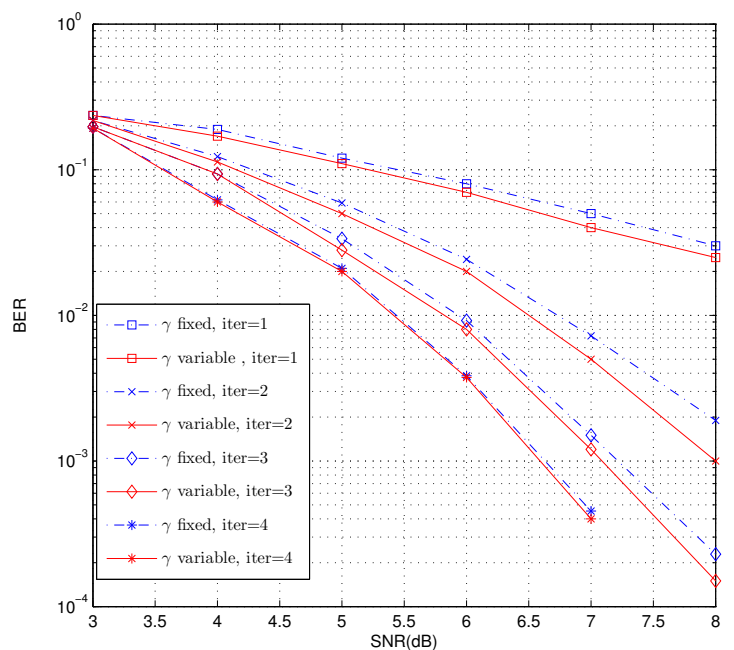

Fig. 6: Influence of $\gamma$ on the BER performance with $N=64$, $n=64, L=512, \mathrm{CC}(13,15)$ and 4-QAM (iterative receiver).

rate performance with a lower complexity compared to $\left(P_{\mathfrak{C}, 2}\right)$. In addition to its asset for the complexity, the detection algorithm output enables to compute a reliable soft input for a FEC decoder. We have also designed an iterative receiver based on a regularization method in the detection.

\section{REFERENCES}

[1] Y. Fadlallah, A. Aissa-El-Bey, K. Amis, D. Pastor, and R. Pyndiah, "New iterative detector of MIMO transmission using sparse decomposition," IEEE Transactions on Vehicular Technology, vol. PP, no. 99, pp. 1-1, 2014.

[2] B. Panzner, W. Zirwas, S. Dierks, M. Lauridsen, P. Mogensen, K. Pajukoski, and D. Miao, "Deployment and implementation strategies for massive MIMO in 5g," in 2014 Globecom Workshops (GC Wkshps), Dec 2014, pp. 346-351.

[3] L. Lu, G. Li, A. Swindlehurst, A. Ashikhmin, and R. Zhang, "An overview of massive MIMO: Benefits and challenges," IEEE Journal of Selected Topics in Signal Processing, vol. 8, no. 5, pp. 742-758, Oct 2014.

[4] A. Aissa-El-Bey, D. Pastor, S. Sbai, and Y. Fadlallah, "Sparsity-based recovery of finite alphabet solutions to underdetermined linear systems," IEEE Transactions on Information Theory, vol. 61, no. 4, pp. 20082018, April 2015.

[5] Y. Fadlallah, A. Aissa-El-Bey, K. Amis, D. Pastor, and R. Pyndiah, "New decoding strategy for underdetermined MIMO transmission using sparse decomposition," in 2013 Proceedings of the 21st European Signal Processing Conference (EUSIPCO), Sept 2013, pp. 1-5.

[6] L. Bahl, J. Cocke, F. Jelinek, and J. Raviv, "Optimal decoding of linear codes for minimizing symbol error rate (corresp.)," IEEE Transactions on Information Theory, 1974.

[7] M. Grant and S. Boyd, "CVX: Matlab software for disciplined convex programming, version 2.1," http://cvxr.com/cvx, Mar. 2014.

[8] I. Gurobi Optimization, "Gurobi optimizer reference manual," 2015. [Online]. Available: http://www.gurobi.com

[9] E. D. Andersen, C. Roos, and T. Terlaky, "On implementing a primaldual interior-point method for conic quadratic optimization," MATHEMATICAL PROGRAMMING SER. B, vol. 95, no. 95, pp. 249-277, 2000.

[10] B. Hassibi and H. Vikalo, "On the sphere-decoding algorithm i. expected complexity," IEEE Transactions on Signal Processing, vol. 53, no. 8, pp. 2806-2818, Aug 2005.

[11] C. Laot, R. L. Bidan, and D. Leroux, "Low-complexity MMSE turbo equalization: a possible solution for edge," IEEE Transactions on Wireless Communications, vol. 4, no. 3, pp. 965-974, 2005. 